\title{
Multiple dental anomalies accompany unilateral disturbances in abducens and facial nerves: A case report
}

Elham Talatahari ${ }^{1,2^{*}}$, Karim Osouli-Bostanabad ${ }^{3}$

1. Department of Pediatric Dentistry, Faculty of Dentistry, Tabriz University of Medical Sciences, Tabriz, Iran

2. Dental and Periodontal Research Center, Tabriz University of Medical Sciences, Tabriz, Iran

3. Azerbaijan Composite Technologists Company, Technology incubator of University of Tabriz, University of Tabriz, Tabriz, Iran

\begin{abstract}
This article describes the oral rehabilitation of an 8-year-old girl with extensively affected primary and permanent dentition. This report is unique in which distinct dental anomalies including enamel hypoplasia, irregular dentin formation, taurodontism, hpodontia and dens in dente accompany unilateral disturbance of abducens and facial nerves which control the lateral eye movement, and facial expression, respectively.
\end{abstract}

Keywords: enamel hypoplasia; irregular dentin formation; taurodontism; hypodontia; dens in dente; abducens and facial nerves;

\section{Introduction}

Tooth development results from a dynamic interplay between regulatory and structural genes and environmental factors which imply the possible vulnerability of these tightly genetically controlled mechanisms to internal and external insults $(1,2)$. Dependent on the time of insult and its association to the developmental stage of the teeth, different disturbing factors can lead to some clinical phenotypes. Varied types of insults can also lead to responses modulating growth and development which in turn can possibly affect the development of the structure and function of the nervous system. If these disturbing factors simultaneously render their effects, dental anomalies can accompany nervous system dysfunction as seen in Möbius syndrome (3), cerebral palsy and Sturge-Weber syndrome (4). This case report presents a rare condition of unilateral disturbance of facial and abducens nerves with distinct dental anomalies.

\section{Corresponding author:}

Elham Talatahari

Department of Pediatric Dentistry, Faculty of Dentistry, Tabriz University of Medical Sciences, Tabriz, Iran PO Box: 5165665931, Iran Tel: +984133355965-9 Fax: +984133346977 E-mail: e_talatahari@yahoo.com

Receive date: 2015-10-06 | Accept date: 2015-11-25 | Publish date: 2015-12-01

DOI: 10.7575/aiac.abcmed.16.04.01.09 
a)

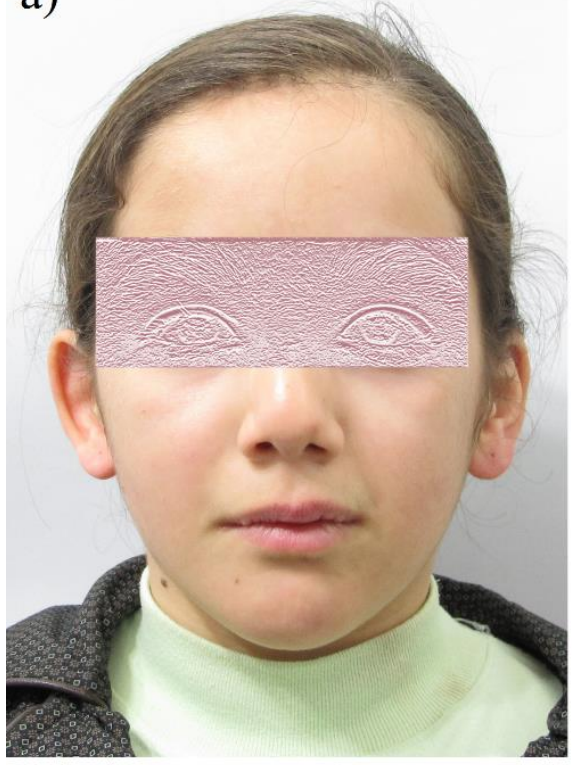

c)

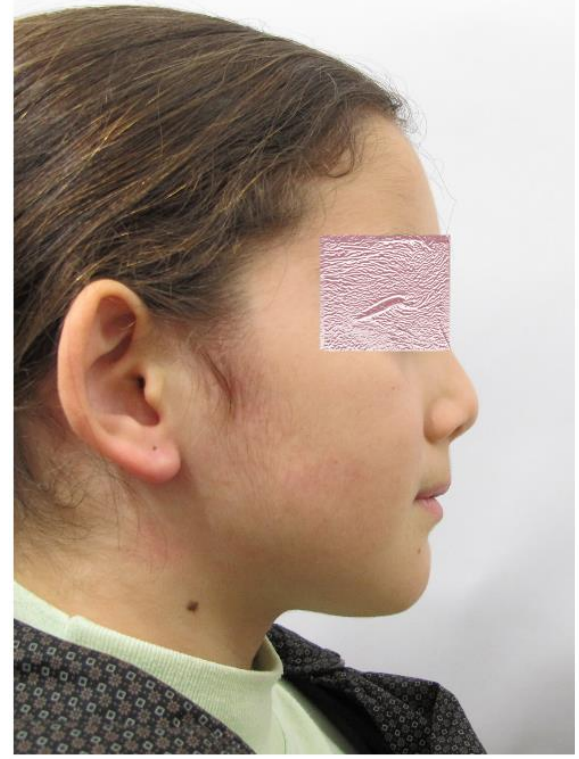

b)

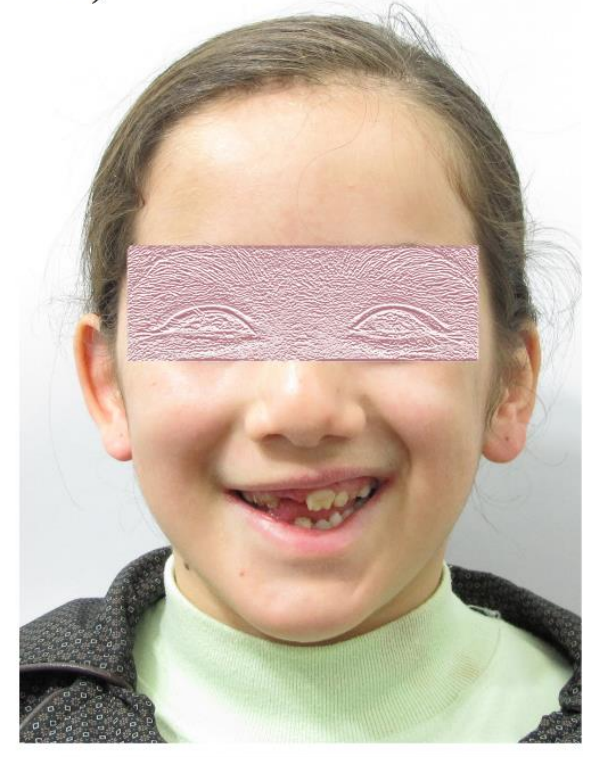

d)

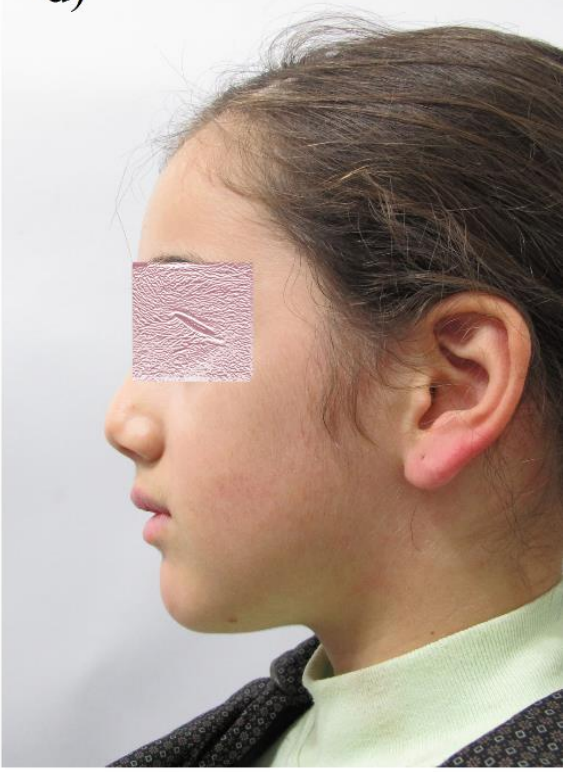

Figure 1: Frontal view of the patient a) at rest, b) at smile and c, d) lateral views of the patient

\section{Case presentation}

An 8 year old female with good socioeconomic status was referred to the postgraduate department of pediatric dentistry, Tabriz University of Medical Sciences, Tabriz, Iran, with the complaint of poor esthetics, discoloration and sensitivity of the anterior teeth and sensitivity of left first permanent molar. The medical history revealed the unilateral facial paralysis in right side (Figure 1). Also, patient had been unable to move the right eye from side to side for which had gone under eye surgery 4 years ago; although, this feature was not evident at this time, it implies the possible disturbance of the VI cranial nerve. The parents reported no similar case in the family. 

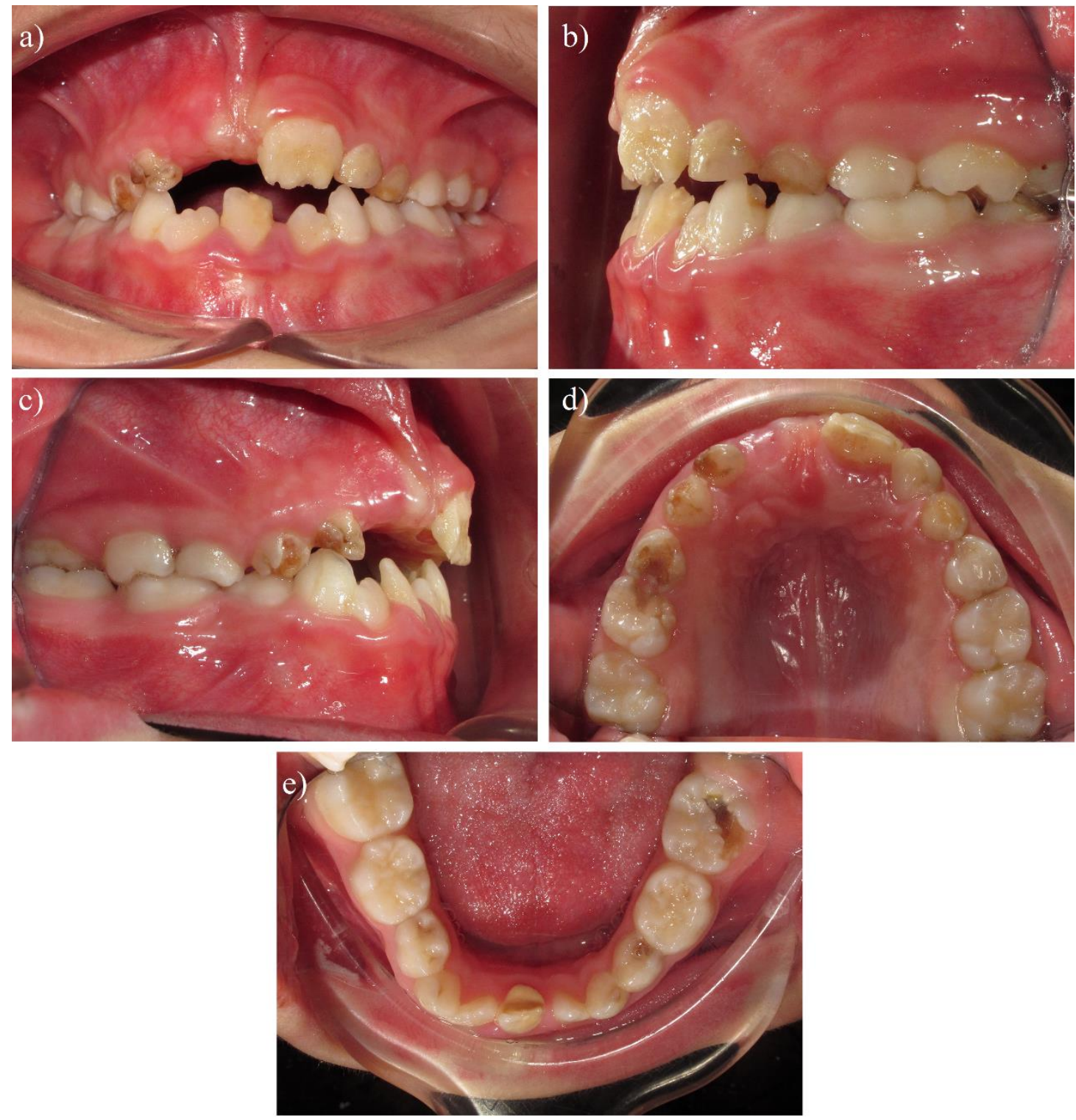

Figure 2: Intra-oral views before treatment. a) Frontal view, b) left lateral view, c) right lateral view, d) upper occlusal view, e) lower occlusal view

On clinical evaluation, teeth were in mixed dentition stage with permanent molars in Angle's class I relationship. Patient had a normal complement of teeth except that upper right permanent second premolar, upper left permanent canine and second premolar were missing. The thickness of enamel was reduced on upper left permanent central incisor, lower right permanent central incisor and lower right first permanent molar and enamel was chipped off from some areas of the teeth exposing the dentin. The surfaces of these affected teeth

were rough. Also, upper left permanent central incisor showed signs of dens in dente. Taurodontism was evident in all first permanent molars except lower left one. In primary dentition, upper right and left first and second molars and lower right and left second molars were affected. Irregular dentinogenesis manifested as abnormal root, root canal and pulp chamber morphology, was obvious in all primary molars (Figure 2, 3).

The treatment plan was directed based on patient's age and needs. The preventive aspect

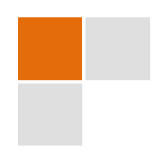




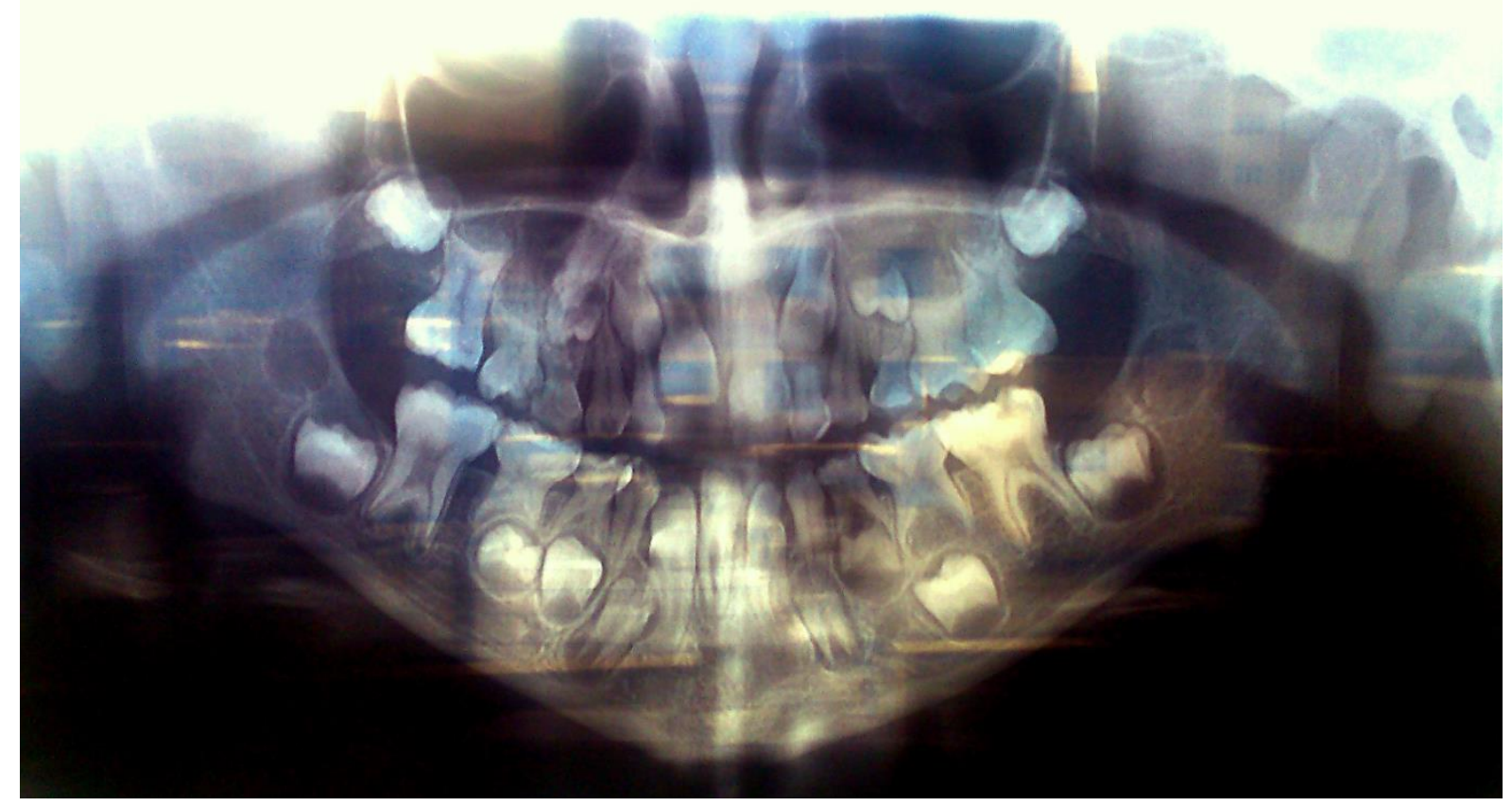

Figure 3: Panoramic radiography of the patient before treatment

included diet and parent counseling and oral prophylaxis using polishing brush. Fluoride therapy was performed with $\mathrm{NaF}$ topical gel (Sultan Healthcare Inc., Englewood, New Jersey, USA) containing the standard $2 \%$ fluoride as discussed by Knutson (5). The original Knutson technique consisted of a series of four applications provided at approximately 1-week intervals, with only the first application preceded by a prophylaxis. lower Left first permanent molar was restored with preformed stainless steel crowns (3M, Unitek, USA) of appropriate size. Crown preparation was done by minimal proximal reduction and odontoplasty of the enamel peaks of the occlusal surface. The crown was cemented using a luting resin modified glass ionomer cement (3 M ESPE, USA) after confirmation in occlusal relations. Upper right first and second primary molars underwent optimal pulp therapies. Then, upper right first and second primary molars and lower left first primary molar received preformed stainless steel crowns (3M, Unitek, USA) of appropriate sizes in the same procedure as explained before.

After considering the age of the patient, the anterior affected teeth were decided to be restored with direct composite veneering using deproteinization technique. The teeth were prepared, conditioned by rubbing sodium hypochloride $5.25 \%$ for 1 minute and rinsed. Acid etching was done using 37\% phosphoric acid (Scotchbond, Dentsply, USA) for 15 seconds. The single bottle adhesive (Prime and Bond NT, Dentsply Caulk) was applied and light cured for 40 seconds. Resin veneering (Esthet-X, Dentsply) was done by appropriate shades using stratified layer technique. Upper left first primary molar and lower right first primary molar received preventive resin restorations (Figure 4) and monthly follow ups scheduled to monitor the efficacy of the treatments and evaluation of the other teeth which will erupt.

\section{Discussion}

This case report presents a rare condition of unilateral disturbance of facial and abducens 

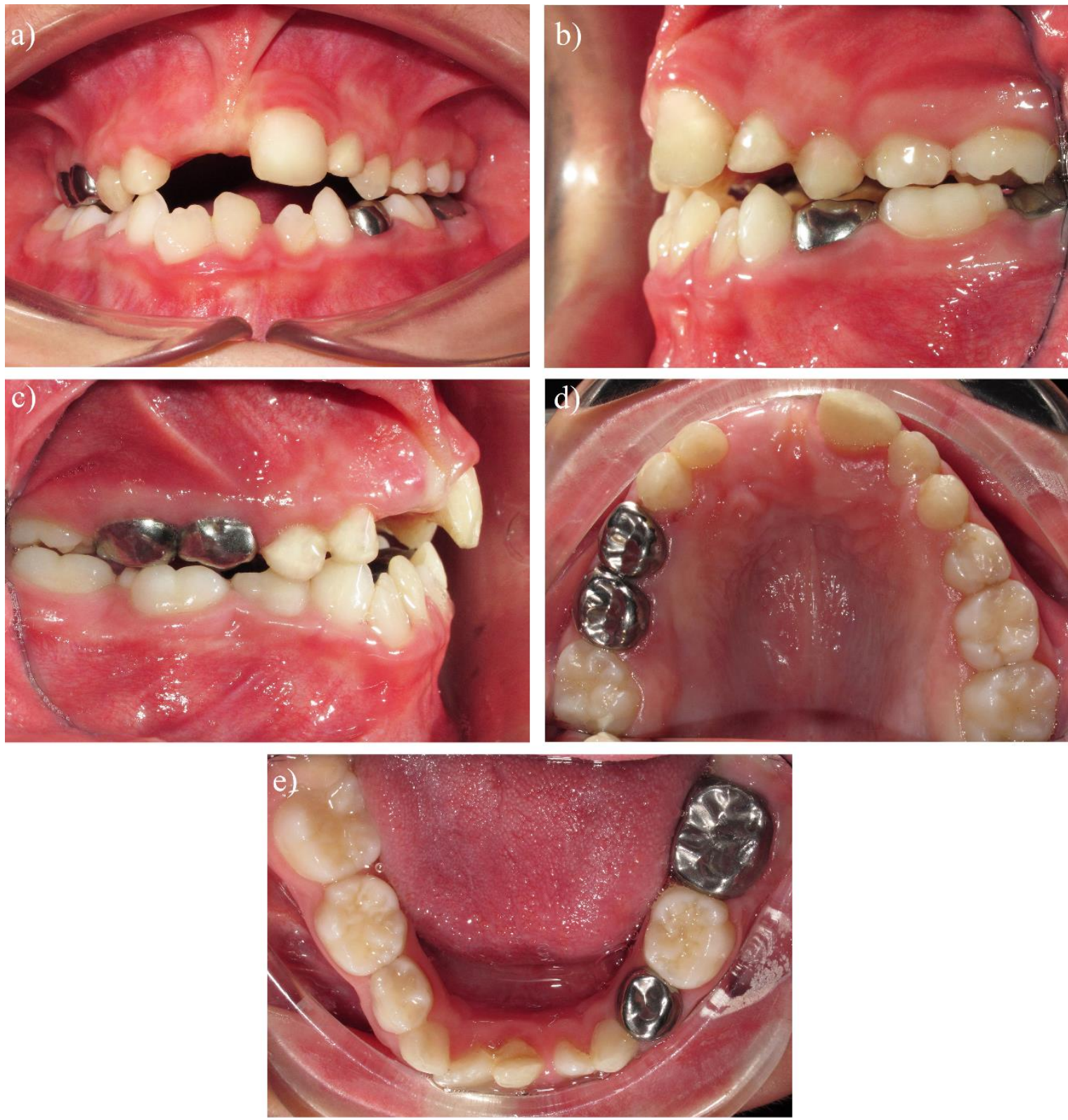

Figure 4: Intra-oral views after treatment. a) Frontal view, b) left lateral view, c) right lateral view, d) upper occlusal view, e) lower occlusal view

nerves with distinct dental anomalies. The patient showed unilateral facial nerve paralysis in right side and history of inability to move the right eye from side to side which resembles some manifestations of Möbius syndrome. This syndrome is a congenital disease resulting from the underdevelopment of the VI and VII cranial nerves (3) which control the lateral eye movement, and facial expression, respectively and have tooth anomalies as an accompanying feature (6). Although the Möbius syndrome can be considered as a differential diagnosis, there are significant differences which distinguish these two conditions such as absence of Limb and chest abnormalities and strabismus which are present in Möbius syndrome and presence of several dental abnormalities other than enamel hypoplasia and missing. Also this syndrome have some facial characteristics such as expressionless face, retraction of the upper lip due to muscle shrinkage, difficulty in breathing and/or in swallowing, frequent 
drooling and increased occurrence of the symptoms of autism which cannot be traced in this case $(3,6,7,8)$.

The patient also manifested multiple dental anomalies in both dentitions. Observed dental anomalies in this patient result from interruption in dental developmental stages of initiation (missing teeth), histo-diffrentiation (enamel hypoplasia and irregular dentin formation) and morpho-diffrentiation (Dens in dente, tuarodontism) in related teeth. Also, existence of different chronological formation and calcification times in each type of teeth and extensive disturbances in different tooth groups implies that the disturbing factors were present in the pre-natal and early post-natal period in this patient.

\section{Summary}

This report addressed the oral rehabilitation of a patient with unilateral disturbance of facial and abducens nerves accompanying distinct dental anomalies.

\section{References}

1. Jernvall J, Thesleff I. Reiterative signaling and patterning during mammalian tooth morphogenesis. Mech Dev 2000;15;92(1):19-29. doi:10.1016/S0925-4773(99)00322-6

2. Beckmann H, Jakob H. Prenatal disturbances of nerve cell migration in the entorhinal region: a common vulnerability factor in functional psychoses?. J Neural Transm Gen Sect 1991;84(1-2):155-64. doi: 10.1007/BF01249120

3. Briegel W. Neuropsychiatric findings of Möbius sequence- a review. Clin Genet 2006;70(2):91-7. doi: 10.1111/j.1399-0004.2006.00649.x

4. Jimmy R, Paul S, Henry W, Dennis J. Pediatric Dentistry - Pageburst E-Book on VitalSource, 4th Edition. Pinkham Chapter 7-Pain Perception Control.

5. Knutson, J.W. Sodium fluoride solution:Technique for applications to the teeth. J Am Dent Assoc 1948;36(3):739.

6. Rizos M, Negron RJ, Serman N. Mobius syndrome with dental involvement: a case report and literature review. Cleft Palate Craniofac J 1998;35(3):262-8. doi: 10.1046/j.1365-263X.2002.00402.x

7. Al Kaissi A, Grill F, Safi H, Ben Ghachem M, Ben Chehida F, Klaushofer. Craniocervical junction malformation in a child with Oromandibular-limb hypogenesis-Möbius syndrome. Orphanet J Rare Dis 2007;8(2):2. doi: 10.1186/1750-1172-2-2

8. Gillberg C, Steffenburg S. Autistic behaviour in Moebius syndrome. Acta Paediatr Scand 1989;78(2):314-6. doi: 10.1111/j.1651-2227.1989.tb11076.x. 